\title{
Correlative Imaging of CD34 Positive Peripheral Blood Stem Cells
}

\author{
L. L. Stojanovic*, M. A. Murphy*, D. Motlagh**, J. P. DiOrio* \\ * Baxter Healthcare Corporation, Technology Resources, ** Baxter Healthcare Corporation, Cellular \\ Therapies - Regenerative Medicine, Round Lake, Illinois, 60073 USA
}

Correlative imaging was used to study the morphology and ultrastructure of freshly-isolated CD34+ peripheral blood stem cells obtained following a positive selection procedure using Baxter's Isolex ${ }^{\circledR}$ device. This instrument uses a magnetic immuno-affinity process to purify CD34 positive cells from growth colony stimulating factor (G-CSF)-mobilized mononuclear cell (MNC) preparations that are obtained by apheresis. The selected CD34 positive cell product is heterogenic, containing multiple cell populations, including immature hematopoietic precursor cells, hematopoietic colony-forming cells and endothelial progenitor cells. Mature peripheral blood lymphocytes, monocytes, granulocytes and platelets do not express the CD34 antigen. The density of this antigen is highest on early hematopoietic progenitor cells and decreases as cells mature. The CD34 antigen is absent on fully differentiated hematopoietic cells.

For both Scanning and Transmission Electron Microscopy (SEM, TEM), the cells were fixed in 2\% glutaraldehyde in $0.1 \mathrm{M}$ cacodylate buffer, rinsed in same buffer and processed through a graded alcohol series from $25 \%$ to $100 \%$ ethanol. For SEM, after dehydration they were processed through an ethanol/hexamethyldisilazane (HMDS) series and air-dried out of the HMDS. For TEM, the cells were osmicated $(2 \%)$ prior to the dehydration series and following dehydration were placed in acetone and embedded in Spurr's resin. The SEM samples were processed on polycarbonate membranes and the TEM samples were handled as cell pellets. The SEM samples were coated with palladium and imaged on a JEOL 7600 field emission SEM at $5 \mathrm{kV}$. For TEM, thin sections were stained with uranyl acetate and lead citrate and the grids were imaged on a JEOL JEM-1200EX TEM at $60 \mathrm{kV}$.

For Confocal Laser Scanning Microscopy (CLSM), the washed cells (in test tubes) were fixed with 4\% paraformaldehyde and then stained with wheat germ agglutinin (WGA)-Alexa Fluor ${ }^{\circledR} 488$ and/or CD34 primary antibody with goat anti mouse IgG-Alexa Fluor ${ }^{\circledR} 568$ secondary antibody, each followed by a wash step via centrifugation. Cells were blocked with FBS/PBS and then goat serum prior to CD34 primary antibody incubation, then were pelleted and resuspended with FBS/PBS and HSA prior to centrifuging them onto slides. ProLong ${ }^{\circledR}$ Gold antifade reagent containing DAPI was added and the slides were cover-slipped and imaging performed on a Nikon A1Rsi CLSM at: $405 \mathrm{~nm}$ (DAPI, blue), $488 \mathrm{~nm}$ (wheat germ agglutinin, green), $561 \mathrm{~nm}$ (CD34, red).

A similar size and spherical appearance was seen for the CD34 positive cells by all three imaging methods employed. In Figures 1-2, a similar staining pattern was observed for both CD34 (red) as well as the WGA (green), which also showed some areas of punctuate stain. WGA binds to Nacetylglucosamine and $\mathrm{N}$-acetylneuraminic (sialic) acid residues in the cell plasma membrane and the CD34 antibody binds to surface antigens on Isolex ${ }^{\circledR}$-selected cells [1]. A predominate nucleus (blue) was also seen. In SEM (Figures 3-4), cellular protrusions were also observed. In TEM (Figures 5-6), these protrusions were also noted, along with a characteristic "notch like" nucleus with disperse heterochromatin [2]. 
References:

[1] Molecular Probes/Invitrogen. Wheat Germ Agglutinin Conjugates. Mar 2009. Eugene, OR. [2] J. M. Radley et al., ExpHematol. 27 (1999) 365.
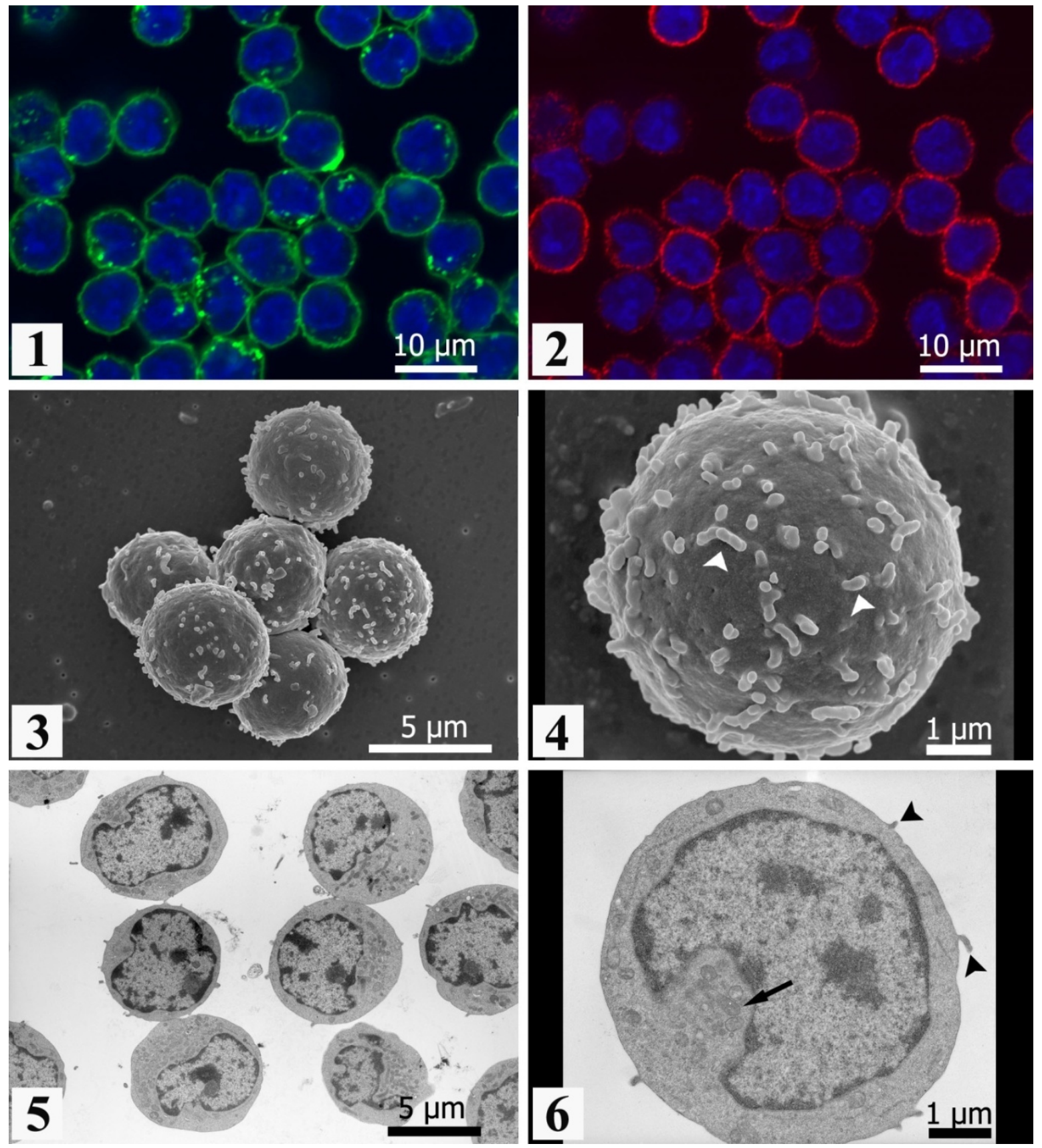

Figures 1, 2: CLSM of CD34 positive cells: blue: nucleus, green: WGA, red: CD34 antigen. Figures 3, 4: SEM of CD34 positive cells: arrow-head at cell protrusions.

Figures 5, 6: TEM of CD34 positive cells: arrow-head at cell protrusions, arrow at mitochondria. 